\title{
Nerve Growth Factor in the Urinary Bladder of the Adult Regulates Neuronal Form and Function
}

\author{
W. D. Steers, ${ }^{\star}$ S. Kolbeck, ${ }^{\star}$ D. Creedon, ${ }^{*}$ and J. B. Tuttle" \\ Departments of ${ }^{*}$ Urology, ${ }^{\ddagger}$ Physiology, and ${ }^{\S}$ Neuroscience, University of Virginia Health Sciences Center, Charlottesville, Virginia 22908
}

\begin{abstract}
Urethral obstruction produces increased voiding frequency $\left(0.7 \pm 0.06\right.$ to $\left.1.1 \pm 0.08 \mathrm{~h}^{-1}\right)$ and hypertrophy of the urinary bladder $(89 \pm 1.7$ to $708 \pm 40 \mathrm{mg})$ with profound increments in the dimensions of afferent $(4,6)$ and efferent neurons $(299 \pm 4.7$ to $573 \pm 8.6 \mu \mathrm{m}^{2}$ ) supplying this organ in the rat. We discovered that hypertrophied bladders of rat and human contain significantly more nerve growth factor (NGF) per milligram wet weight, protein, and DNA than normal bladders. The temporal correlation between NGF content, neuronal hypertrophy, and bladder weight was consistent with a role for this growth factor in the neurotrophic effects associated with obstruction. Autoimmunity to NGF abolished the hypertrophy of NGF-sensitive bladder neurons in the pelvic ganglion after obstruction. Relief of urethral obstruction reduced bladder size ( $349 \pm 78 \mathrm{mg})$, but neuronal hypertrophy $\left(460.2 \pm 10.2 \mu \mathrm{m}^{2}\right)$ and elevated NGF levels were only partially reversed. Bladder hypertrophy $(133 \pm 4.3$ mg) induced by osmotic diuresis slightly increased ganglion cell area $\left(365.2 \pm 6.1 \mu \mathrm{m}^{2}\right)$ and only doubled NGF content of the bladder. These findings provide important new evidence that parenchymal cells in the hypertrophied bladder can synthesize NGF and possibly other molecular messengers that act to alter the size and function of neurons in adult animals and man. ( $J$. Clin. Invest. 1991. 88:1709-1715.) Key words: hypertrophy • outlet obstruction $\bullet$ neural plasticity $\bullet$ smooth muscle $\bullet$ major pelvic ganglion
\end{abstract}

\section{Introduction}

Urinary bladder hypertrophy is produced by anatomical or functional obstruction of the bladder outlet. The most common cause of obstruction in man is benign prostatic hypertrophy $(\mathrm{BPH})^{1}$, the treatment of which represents the second costliest expenditure of Medicare funds in the United States

Part of this work appeared in abstract form (Creedon, D., W. Steers, and J. Tuttle. 1989. Abstr. Soc. Neurosci. 16:301).

Address reprint requests to J. B. Tuttle, Ph.D., Department of Neuroscience, Box 230, University of Virginia Health Sciences Center, Charlottesville, VA 22908.

Received for publication 13 February 1991 and in revised form 27 June 1991.

1. Abbreviations used in this paper: $\mathrm{AI}$, autoimmunization; BPH, benign prostatic hypertrophy; CONT, control; CPRG, chlorophenol redbeta-D-galactopyranoside; DELIG, deligation; DIUR, diuresis, DRG, dorsal root ganglia; FG, Fluoro-Gold; NGF, nerve growth factor; OBS, obstruction; TH-IR, tyrosine hydroxylase.

J. Clin. Invest.

(c) The American Society for Clinical Investigation, Inc.

0021-9738/91/11/1709/07 $\$ 2.00$

Volume 88, November 1991, 1709-1715
(1). A manifestation of bladder dysfunction seen in $60-75 \%$ of patients with BPH is bladder hyperactivity (2). Because obstruction of the bladder leads to changes in neuronal morphology and function, these alterations in neural pathways could contribute to bladder dysfunction and alter treatment outcome (3-7).

It has been proposed that dynamic interactions occur between a target tissue and its innervation in the adult $(8,9)$. In support of this idea experimental manipulations of a target alter the geometry of innervating neurons. For example, creating large targets results in more morphological complexity in neural pathways, whereas reductions in target size produce smaller, less complex neurons $(8,9)$. This interaction between a tissue and its nerve supply has been attributed to the action of as yet unidentified molecular messengers. One candidate that fits criteria for an intercellular signal molecule between the target organ and nerve is nerve growth factor (NGF)(10). However, a specific role for NGF in the regulation of neural plasticity in vivo or in a disease process in the adult has not been established.

The accessibility of peripheral autonomic neurons provides an opportunity to perform a detailed analysis of the relationships between neuronal geometry and the function or size of the target innervated. One target organ whose function requires autonomic input is the urinary bladder. Thus, bladder hypertrophy induced by partial obstruction may offer a clinically relevant model that provides insight into neurotrophic interactions in vivo.

Indeed, partial urethral ligation in the rat, which produces bladder enlargement and smooth muscle hypertrophy, is associated with morphological and physiological alterations in the neural pathways regulating micturition. Morphological changes include hypertrophy of retrogradely labeled afferent $(5-7)$ and efferent $(4,7)$ neurons supplying the bladder, as well as increased labeling of afferent projections from the bladder surrounding preganglionic neurons in the sacral spinal cord $(6$, 7). Physiological alterations are seen in multiunit recordings from bladder nerves (3). Enhanced spinal reflex discharges appear after obstruction which are linked to an increase in voiding frequency (3). Decentralization of the bladder fails to prevent the size increases of neurons induced by obstruction (4). Taken together these findings provide strong evidence that neurotrophic interactions occur between the bladder and its innervation and that these interactions are altered after obstruction.

It is proposed that obstruction stimulates parenchymal cells in the bladder to produce a factor which regulates size of the neurons supplying this organ. This hypothesis was tested by assaying normal and hypertrophied bladders for NGF to assess the potential involvement of this single trophic factor on changes in efferent neuron size. We report that increased tissue levels of NGF accompany urinary bladder hypertrophy in rats and humans. Furthermore, endogenous NGF antibody pre- 
vented the hypertrophy of a population of efferent nerves innervating the bladder.

These findings have profound investigational and clinical ramifications. They provide important new evidence for dynamic interactions between parenchymal target cells and their innervation in the adult. It can be speculated that alterations in NGF or other trophic factors participate in the sensory disturbances and bladder hyperactivity observed in patients with BPH, suggesting novel avenues for therapy.

\section{Methods}

\section{Experimental groups}

Obstruction (OBS). A modification of the technique described by Mattiasson and Uvelius $(3,4,11)$ was used to create partial bladder outlet obstruction in $13 \mathrm{Wistar}$ rats $(222-250 \mathrm{~g})$. Under halothane anesthesia, the skin was shaved, prepped with an iodine/alcohol mixture, and a low midline incision made. The urethral diameter was then reduced to 1 $\mathrm{mm}$ by tying two 4-0 nylon sutures around the urethra and an extraluminally-positioned (1 mm O.D.) polyethylene tube. The tubing was removed and the abdominal incision reapproximated using two layers of interrupted nylon sutures. 12 control (CONT) rats underwent sham surgery in which the urethra was circumferentially dissected, but not ligated. Occasionally, a ligated animal developed urinary retention or was incontinent as monitored by voiding frequency, presumably due to overflow incontinence. These animals were sacrificed immediately and not included in the study.

Diuresis (DIUR). Bladder hyperactivity without obstruction was produced in eight rats by inducing osmotic diuresis with $5 \%(\mathrm{wt} / \mathrm{vol})$ sucrose in their ad lib water supply.

Deligation (DELIG). 6 wk after urethral ligation, six rats underwent surgical removal of urethral ligatures under halothane anesthesia to examine reversibility of the changes in voiding frequency, neuron size, and levels of NGF.

Autoimmunization (AI). 30 female rats were immunized with 100 $\mu \mathrm{g}$ murine NGF in complete Freund's adjuvant followed by $15-\mu \mathrm{g}$ injections in incomplete adjuvant at 4-5-wk intervals (12). Successful AI was evaluated in each animal by testing for circulating anti-NGF activity using a modified neurite outgrowth bioassay. Dorsal root ganglia (DRG) from 8-9-d-old chick embryos were cultured in equal 20- $\mu 1$ portions of chicken plasma, thrombin $(1 \mathrm{mg} / \mathrm{ml})$ in modified Eagles medium and diluted rat tail vein serum in culture medium with 15 $\mu \mathrm{g} / \mathrm{ml}$ murine NGF. Positive controls were included with titers of known, purified anti-NGF. Under these conditions fiber outgrowth was profuse when no antiserum was added. Animals were considered autoimmune to NGF if $1 / 100$ to $1 / 1,000$ dilution of serum prevented neurite outgrowth. Neurite outgrowth was evaluated using a Nikon Diaphot inverted microscope and phase contrast optics. Fiber outgrowth was easily distinguished from outgrowth of explant supporting cells.

Autoimmune + obstructed (AIOBS). Six AI animals (see Autoimmunization) were obstructed (see Obstruction) to determine whether endogenous antibody to NGF prevented the neuronal growth and urodynamic changes accompanying obstruction.

Neuronal labeling. Bladder neurons were labeled by retrograde axonal transport of Fluoro-Gold (FG; Fluorochrome, Inc., Englewood, $\mathrm{CO}$ ) because ganglion cells in the major pelvic ganglion supply several organs. Eight separate injections (3-4 $\mu$ ) of $4 \%$ FG (wt/vol in distilled water) were made into the bladder wall using a 10- $\mu$ l Hamilton syringe with a $30 \mathrm{G}$ needle. The needle was left in place for $30 \mathrm{~s}$ and the site rinsed with saline then swabbed with a cotton-tipped applicator to minimize leakage of tracer. FG injection was performed $48 \mathrm{~h}$ before removal of ganglia. Injected bladders were not used for NGF assays.

Human subjects. 2-g specimens of smooth muscle from the anterior bladder wall were taken for NGF analysis from patients $(n=3)$ undergoing suprapubic prostatectomy for obstruction due to prostatism or urinary diversion for functional bladder outlet obstruction resulting from spinal cord injury. Bladders from obstructed subjects were grossly thickened and trabeculated. Voiding flow rates were $<10 \mathrm{ml} / \mathrm{s}$ consistent with obstruction. Obstructive symptoms such as diminished urine flow, nocturia, and hesitancy and irritative symptoms of urinary urgency and frequency were present for at least 1 yr. Control $(n=3)$ specimens were obtained from patients undergoing urinary diversion for incontinence or bladder cancer. Specimens were not obtained from areas which contained malignancy. Control subjects did not have obstructive symptoms or flow rates $<20 \mathrm{ml} / \mathrm{s}$. Tissues were obtained in accordance with the University of Virginia Human Investigation Committee.

\section{Analysis}

Measurements of voiding frequencies, neuronal areas, and NGF content were made in a single blinded fashion with the investigator unaware of which group of animals were being analyzed.

Urinary frequency. Voiding frequency was used as an estimate of bladder activity in the experimental animals. Animals were housed in individual cages. Voiding frequency was assessed in a single blinded fashion on four separate occasions in each animal by visualizing distinct urine markings on filter paper placed beneath each cage during a 3-h test period (16:00-19:00 h) with monitoring of fluid intake. Obstruction did not alter water consumption.

Morphometrics. Pelvic ganglia were removed in halothane-anesthetized rats and placed in $4 \%$ buffered formalin for $4 \mathrm{~h}$. Ganglia were washed in PBS and equilibrated in successive PBS solutions containing $10,20$, and $30 \%$ sucrose for cryoprotection. Cryostat sections (14 $\mu \mathrm{m})$ were cut and mounted on gelatinized slides, dehydrated using a series of alcohols, mounted in glycerin, and cover slipped. FG-labeled ganglia were then viewed using a Nikon FXA microscope with UV fluorescence (filter UV excitation at $360-380 \mathrm{~nm}$ ).

Cross-sectional areas of FG-labeled cells in every fourth section and distributed throughout the pelvic ganglia were measured using computerized image processing (Bioquant) and videomicroscopy. Video images were obtained through a Dage-MTI Inc. (Wabash, MI) 70 series camera at a magnification of $200 . \sim 200$ cells from each ganglion were manually outlined for area determinations. For comparison, cross-sectional areas as measured using camera lucida drawings were $10 \%$ smaller than those obtained through image analysis software. The lower limit for neuronal cross-sectional areas was set at $150 \mu \mathrm{m}^{2}$ based on previous measurements of principal cells in the major pelvic ganglion (4).

Tissue assays. Urinary bladders and other tissues were removed under halothane anesthesia, blotted dry, weighed, frozen on dry ice, and stored at $-70^{\circ} \mathrm{C}$. For analysis, tissues were shattered at liquid nitrogen temperature in a metal impact mortar, and solubilized in $4 \mathrm{vol}$ or a minimum of $1-\mathrm{ml}$ sample buffer $\left(0.1 \mathrm{M} \mathrm{NaHPO}_{4}, 0.4 \mathrm{M} \mathrm{NaCl}\right.$, $0.1 \%$ Triton $\mathrm{X}-100,0.5 \%$ BSA, $0.1 \mathrm{mM}$ benzethonium- $\mathrm{Cl}, 2 \mathrm{mM}$ benzamidine, $2 \mathrm{mM}$ EDTA, $0.1 \mathrm{mM}$ PMSF, $20 \mathrm{KIU} / \mathrm{ml}$ aprotinin). The tissue was then homogenized for $1 \mathrm{~min}$ and then centrifuged at 33,000 $g$ for $1 \mathrm{~h}$. To estimate NGF recovery, $150 \mathrm{pg} / \mathrm{ml}$ of authentic NGF was added to an aliquot before the centrifugation. Aliquots of the total homogenate were also assayed for protein (13) and DNA (14).

A two-site ELISA for NGF was adapted from the methods of Westcamp and Otten (15) and Hellweg (16). Maxisorb 96-well plates (Nunc, Copenhagen, Denmark) were coated with $150 \mu \mathrm{l}$ of $0.5 \mu \mathrm{g} / \mathrm{ml}$ antiNGF monoclonal antibody (Boehringer Mannheim Biochemicals, Indianapolis, IN) in $50 \mathrm{mM}$ of sodium carbonate buffer, $\mathrm{pH} 9.6$, for $2 \mathrm{~h}$ at $36^{\circ} \mathrm{C}$. Remaining sites on the plate were blocked with $200 \mu \mathrm{l}$ of a $1.0 \%$ BSA in a carbonate buffer at $1 \mathrm{~h}$ at $36^{\circ} \mathrm{C}$. The plate was then washed 1 $\times 1 \mathrm{~min}, 3 \times 5 \mathrm{~min}$ with $250 \mu \mathrm{l}$ of wash buffer $(0.4 \mathrm{M} \mathrm{NaCl}$ and $0.1 \%$ Triton X-100 in 0.1 M phosphate buffer, pH 7.4). The final sample was replaced with $100 \mu \mathrm{l}$ of either sample or known amounts of NGF diluted in sample buffer. Four wells were used for each sample and for each value of the standard curve. The samples were incubated overnight at room temperature in a humidified chamber. The following day the plate was washed $1 \times 1 \mathrm{~min}, 3 \times 20 \mathrm{~min}$ with slow agitation. To each well, $10 \mathrm{mU}$ of $\beta$-galactosidase-linked anti-NGF (Boehringer 


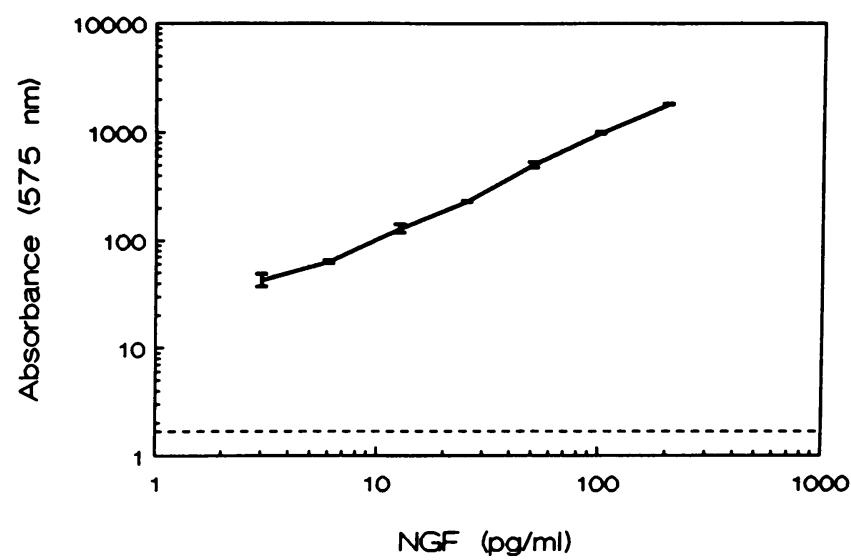

Figure 1. Standard curve for NGF ELISA. Plot was linear to $1 \mathrm{pg} / \mathrm{ml}$ of NGF. Mouse NGF was used to construct standard curve. Absorbance obtained at $575 \mathrm{~nm}$. Broken line represents three standard deviations from blank specimen.

Mannheim Biochemicals), was then added for $2 \mathrm{~h}$ at $36^{\circ} \mathrm{C}$. Unbound $\beta$-galactosidase was removed during $1 \times 1 \mathrm{~min}, 3 \times 1 \mathrm{~h}$ washes in buffer with intermittent agitation. During the last buffer wash, $44 \mathrm{mg}$ of chlorophenol red-beta-D-galactopyranoside (CPRG; Boehringer Mannheim Biochemicals) was dissolved in $22 \mathrm{ml}$ of $10 \mathrm{mM}$ Hepes, $150 \mathrm{mM}$ $\mathrm{NaCl}, 2 \mathrm{mM} \mathrm{MgCl}, 0.1 \%$ (wt/vol) Na-azide, and $1 \%$ (wt/vol) BSA, pH 7.0. The plate was rinsed once with $250 \mu \mathrm{l}$ of CPRG buffer and $200 \mu \mathrm{l}$ of the CPRG solution was added for $4-6 \mathrm{~h}$ at $36^{\circ} \mathrm{C}$ or overnight at room temperature. The plate was read on a Titretek plate reader (ICN/Flow Laboratories, Inc., McLean, VA) using a 575-nm filter.

The standard curve was calculated after subtracting the mean absorbance reading of sample buffer alone based upon a least squares fit (Fig. 1). Any value falling within three standard deviations of the zero NGF reading was considered below the limit of detection, typically occurring at $<1 \mathrm{pg} / \mathrm{ml}$ (Fig. 1).

The mouse NGF used for the standard curve was the generous gift of Dr. Eugene M. Johnson (St. Louis, MO), and was freshly diluted in sample buffer for each assay. Controls for the assay always included binding nonimmune mouse IgG to the plate and the zero NGF point on the standard curve. Specificity for antibody binding to NGF was tested with insulin, a structurally similar molecule of a similar size. The standard curve for NGF typically was linear to $600 \mathrm{pg} / \mathrm{ml}$, beyond which saturation occurred. However, $1 \mu \mathrm{g} / \mathrm{ml}$ insulin was below the level of detection. The absorbance values for the standards varied, but the slope of the standard curve was consistent. The specificity of the assay for NGF compared to other neurotrophins in the same family (BDNF and NT3) could not be assayed due to lack of sufficiently characterized reagents. Thus, the tissue samples may have contained some levels of cross-reactive factors structurally similar to NGF.

Human tissues were assayed by W. Wong and G. Bennett at Genentech, Inc. (South San Francisco, CA) using a similar two-site ELISA but with antiserum specific for human NGF. A standard curve was obtained using recombinant human NGF and an horseradish peroxidaselinked secondary antibody.

Statistics. In all studies mean cross-sectional areas for FG-labeled neurons were compared using an ANOVA while voiding frequencies, bladder weights, and NGF content were analyzed with a nonparametric Mann/Whitney U test using SPSS PC+ software. Differences in the distributions of neuronal areas were analyzed using the Smirnoff test. Data presented as mean $\pm \mathrm{SE}$.

\section{Results}

Bladder weight increased from $89 \pm 1.7 \mathrm{mg}$ in the control group to $708 \pm 40 \mathrm{mg}$ after $6 \mathrm{wk}$ of obstruction (OBS) (Fig. $2 \mathrm{~A}$ ). The average frequency of urinary voiding increased from $0.7 \pm 0.06$ to $1.1 \pm 0.08 \mathrm{~h}^{-1}$ after obstruction confirming the development of bladder hyperactivity (Fig. $2 \mathrm{~A}$ ). The mean cross-sectional area of pelvic ganglion cells innervating the obstructed bladder rose significantly reaching $573.0 \pm 8.6 \mu \mathrm{m}^{2}$ at $6 \mathrm{wk}(P<0.001)$ (Fig. $2 A$ ). Neuronal hypertrophy was detected after 1 wk, lagging behind the rapid rise in bladder weight.

Bladder enlargement was accompanied by a rise in NGF content which preceded increases in voiding frequency and neuronal area (Fig. 2). Likewise, the amount of NGF in grossly

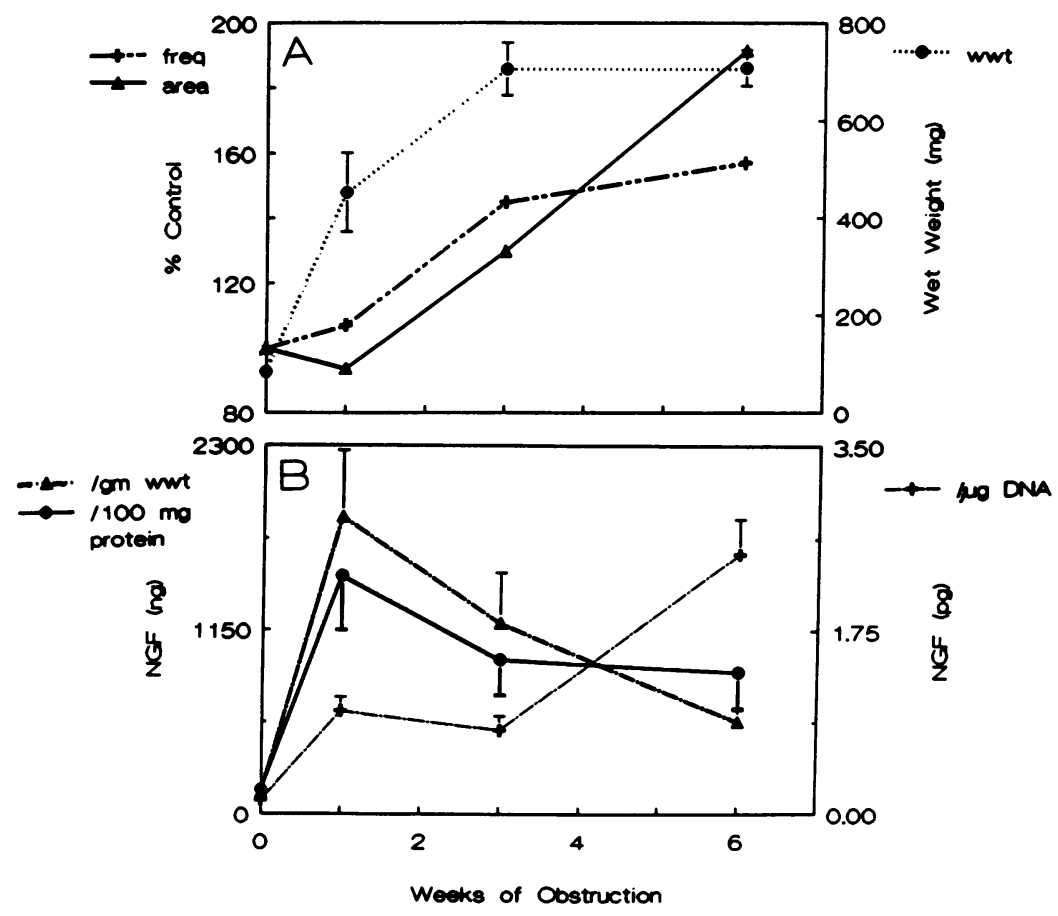

Figure 2. Time course for alterations in bladder weight, function, and neuronal size after obstruction. Bladder weight in milligrams (๑), whereas voiding frequencies $(+)$ and neuronal areas $(\Delta)$ are expressed as percent control $\left(0.7 \pm 0.05 \mathrm{~h}^{-1}, 300 \pm 4.9 \mu \mathrm{m}^{2}\right.$ for 750 cells). Neuronal size was measured as cross-sectional area of retrogradely labeled pelvic ganglion cell somata at $1 \mathrm{wk}$ ( 663 cells), $3 \mathrm{wk}$ ( 575 cells), and $6 \mathrm{wk}$ (750 cells). Bladder weight rises before the increases in voiding frequency and neuron size. $(B)$ Bladder tissue NGF. NGF is expressed as (•) nanograms per $100 \mathrm{mg}$ protein of the whole homogenate; $(\Delta)$ nanograms per gram wet weight; and (+) picograms per microgram DNA. NGF expressed per wet weight or protein peaks before changes in neuronal area or voiding frequency. 
hypertrophied human bladders $(7.8 \mathrm{pg} / \mathrm{mg}$ pro, $1400 \mathrm{pg} / \mathrm{g}$ wet weight) exceeded that in nonhypertrophied samples $(2.6 \mathrm{pg} / \mathrm{mg}$ pro, $400 \mathrm{pg} / \mathrm{g}$ wet weight) from the patients undergoing urinary diversion for incontinence.

Tissue levels of NGF were assayed in organs other than the urinary bladder from normal and obstructed animals to ascertain whether the increases in bladder NGF were specific for the urinary tract. No significant differences were seen in NGF content for abdominal aorta or lung specimens (CONT, $n=5$; OBS, $n=6$ ). NGF was not detected in striated or cardiac (ventricular) muscle samples.

If NGF is responsible for one or more aspects of the neuronal plasticity after bladder obstruction and hypertrophy, levels of this trophic factor should rise before increases in neuron size and voiding frequency. Indeed, the amount of NGF peaked 1 wk after obstruction whether the data are expressed per whole organ, milligram protein, or gram wet weight of bladder tissue (Fig. 2 B). Over the ensuing weeks NGF levels dropped below peak values but remained four to six times higher than control values. When normalized to DNA, NGF content continued to rise after 3 wk (Fig. 2 B).

After deligation, neuronal size $\left(460.2 \pm 10.2 \mu \mathrm{m}^{2}\right)$ and NGF levels failed to return to control values (Fig. 2), despite the significant $(P<0.05)$ decrease in bladder weight $(349 \pm 78 \mathrm{mg})$ and return of voiding frequency to CONT levels $(0.73$ $\pm 0.08 \mathrm{~h}^{-1}$ ).

In rats with bladder hypertrophy induced by diuresis, bladder weight $(133 \pm 4.3 \mathrm{mg})$, voiding frequency $\left(1.0 \pm 0.09 \mathrm{~h}^{-1}\right)$, neuronal area $\left(365.2 \pm 6.1 \mu \mathrm{m}^{2}\right)$, and NGF levels were elevated compared to control. Yet, weights, areas, and NGF levels from the diuresis group were significantly $(P<0.01$ to $P<0.001)$ less than those in the OBS group.

The temporal correlation between NGF, neuronal hypertrophy, and bladder weight does not establish NGF as the causative factor for neurotrophic effects. Thus, we sought to obtain more conclusive evidence by using endogenous antibody against NGF which is effective in disrupting the access of neurons to the nutritive supply of this trophic substance $(10,12)$. Bladder weights in the AI group were slightly greater $(116 \pm 4$ $\mathrm{mg}$ ) than control. Despite this slight increase in bladder weight, the mean area for neurons innervating the bladder from animals with endogenous antibody against NGF (AI) (Fig. 3) was less $\left(279.3 \pm 3.4 \mu \mathrm{m}^{2}, P<0.05\right)$ than age and weight-matched control $\left(300.2 \pm 4.9 \mu \mathrm{m}^{2}\right)$. The percent of neurons with areas $>500 \mu \mathrm{m}^{2}$ was substantially reduced when obstruction was created in the AI group (Fig. 4, AIOBS-OBS). This inhibition accounted for the reduced mean area for the AIOBS group $\left(475.7 \pm 6.5 \mu \mathrm{m}^{2}\right)$ compared to OBS animals with no circulating anti-NGF $\left(573 \pm 8.6 \mu \mathrm{m}^{2}, P<0.001\right)$. Bladder weights in the AI-OBS group $(800 \pm 65 \mathrm{mg}$ ) were similar to OBST.

In contrast to areas, voiding frequency in AI rats was elevated $\left(1.14 \pm 0.08 \mathrm{~h}^{-1}, P<0.05\right)$ compared with control. This increase in voiding frequency may have accounted for the slight increase in bladder weights in the AI group. Obstruction of $\mathrm{AI}$ animals elicited a further increase in voiding frequency $(1.8 \pm 0.1)$ which exceeded that of the OBS group $(P<0.05)$.

\section{Discussion}

Our results support the concept that the geometry of autonomic neurons in the adult is influenced by ongoing interac-

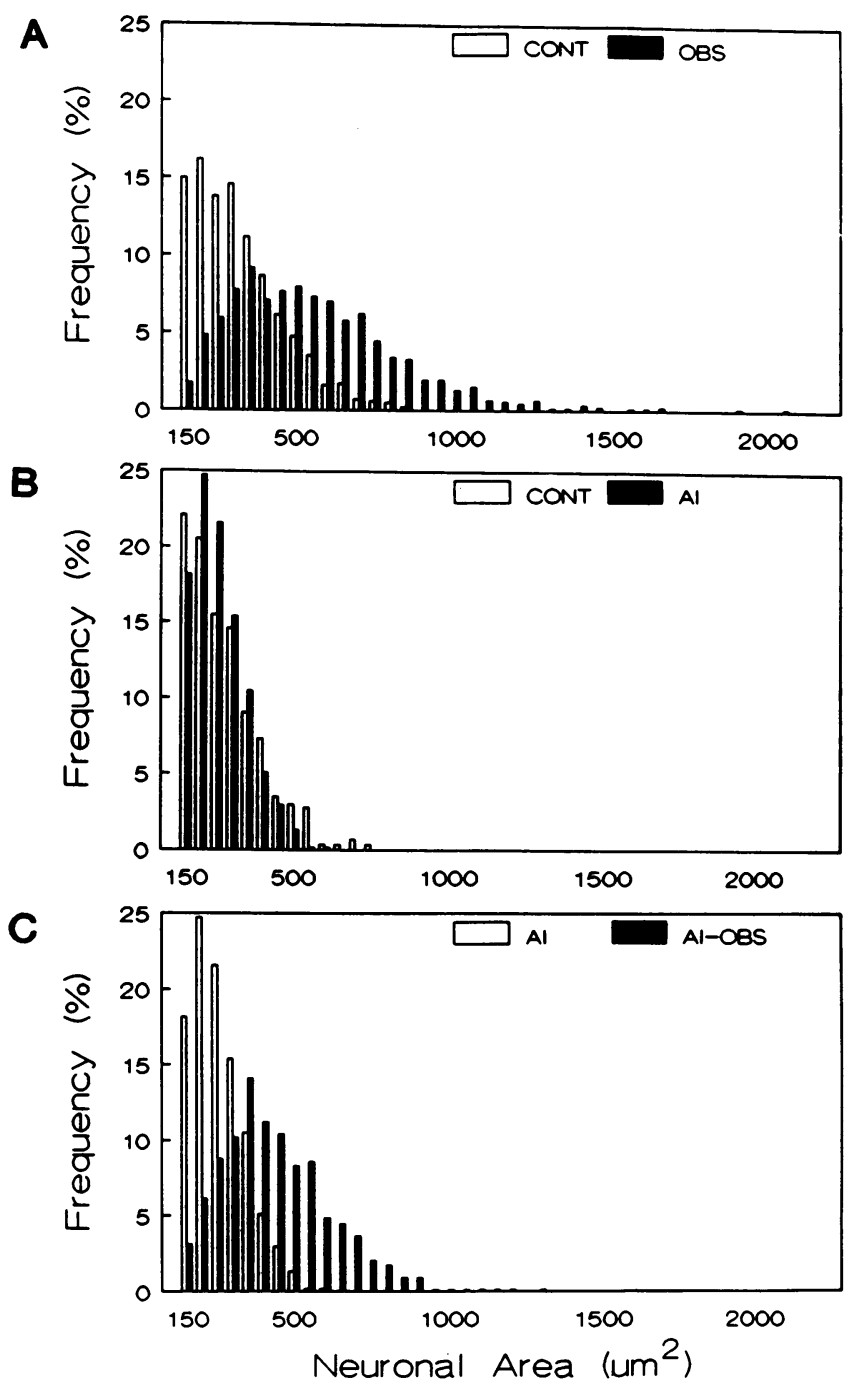

Figure 3. Area distributions for retrogradely labeled bladder neurons in the pelvic ganglia in normal (CONT), obstructed $(O B S)$, autoimmune $(A I)$, and $\mathrm{AI}+\mathrm{OBS}$ animals. $(A) 6 \mathrm{wk}$ after partial urethral ligation an increase in mean area $\left(573 \pm 8.6 \mu \mathrm{m}^{2}\right.$ for 750 cells) was due to a rightward shift in distribution for cell sizes. Note that the entire population shifts with the emergence of cells with areas $>1,000 \mu \mathrm{m}^{2}$. (B) Autoimmunization produces a loss of labeled neurons (611 cells) with areas $>800 \mu \mathrm{m}^{2}$. (C) After 6 wk of obstruction of AI rats (715 cells), the occurrence of cells in the largest size categories is reduced compared to $A$ and neurons rarely have areas $>1,000 \mu \mathrm{m}^{2}$.

tions with the tissues they innervate $(8,9)$. Consistent with this hypothesis, obstruction-induced hypertrophy of the small intestine stimulates growth of neurons in the myenteric plexus $(17,18)$. Likewise, Voyvodic has shown hypertrophy of the submandibular gland increases somal cross-sectional areas and creates more complex dendritic arborization of innervating neurons (9).

Similarly the urinary bladder undergoes anatomical, biochemical, pharmacological, and physiological changes in response to the functional demands of chronic obstruction (2-4, $11,19)$. These changes are accompanied by growth of afferent and efferent neurons supplying the urinary bladder which may contribute to dysfunction and also to the failure of therapy to reverse symptoms. The results of this investigation raise the 


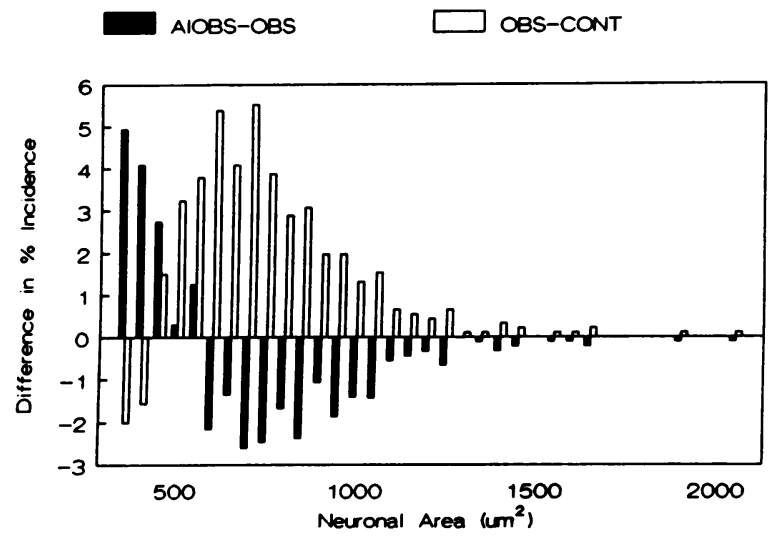

Figure 4. Differences in the frequencies of bladder neuron size in the pelvic ganglion with obstruction and NGF autoimmunity. The differences in percent neurons in each $50 \mu \mathrm{m}^{2}$ bin are plotted for obstructed $(O B S)$ minus control (CONT) frequencies (open bars) and NGF autoimmune obstructed $(A I O B S)$ minus obstructed $(O B S)$ frequencies (solid bars). A positive value indicates an increase in the proportion of cells in that bin, whereas a negative value represents a decrease. Obstruction (OBS-CONT) increases the proportion of large neurons with areas $>500 \mu \mathrm{m}^{2}$. However, AIOBS animals have a relative deficiency in the largest sizes $(A I O B S-O B S)(P<0.001)$. This implies that antibody prevented hypertrophy of NGF-responsive neurons.

possibility that the bladder uses NGF to initiate or maintain these alterations in neural pathways. Whereas the current data only address size changes in the MPG, adult rat DRG neurons have been shown to respond to NGF (12). Additional studies are underway to examine the effect of increased bladder NGF on central afferent projections and electrophysiological alterations after obstruction of the bladder outlet.

NGF content in the bladder peaked 1 wk after obstruction, before hypertrophy of bladder neurons. When normalized to DNA content, NGF continued to rise after 3 wk of obstruction. These findings suggest that the initial rise in NGF was outpaced by bladder hypertrophy. The number of neurons innervating the obstructed bladder is constant (4), but this enlarged target organ could provide an excess of a trophic molecule to each ganglion cell. Thus, the obstructed bladder may deliver up to 50 times more NGF to each ganglion cell than a normal bladder based upon NGF content in the total organ and ignoring possible differences in access and transport (20). Neuron hypertrophy could occur in response to this rapid increase in the peripheral supply of NGF.

The temporal correlation between bladder weight, NGF content, neuronal hypertrophy, and changes in bladder function are consistent with a link between this trophic molecule and adaptive changes in neural pathways with obstruction. However, the strongest evidence that NGF is at least in part responsible for these alterations was the finding that endogenous antibody to NGF reduced the proportion of cells in the uppermost size categories for neurons supplying the bladder after obstruction.

In dissociated cell culture, pelvic ganglion cells from the rat immunoreactive for tyrosine hydroxylase (TH-IR) are eliminated by NGF antibody (21). Neurons in the pelvic ganglion innervating the bladder with areas $>500 \mu \mathrm{m}^{2}$ are TH-IR (22). Furthermore, administration of NGF antibody to neonatal mice eliminates catecholamine-fluorescent varicosities in pel- vic ganglia (23). These observations imply that NGF antibody in vivo probably affects adrenergic neurons in the major pelvic ganglion. It is unlikely that the effects of obstruction are merely limited to adrenergic neurons because a shift in area occurs for the entire population of bladder neurons (4) and TH-immunoreactive neurons account for $\sim 25 \%$ of the cells in the pelvic ganglion (22).

Muscle activity in addition to target size has been shown to alter neuron survival and morphology. Thus, an increase in contractile activity of the bladder due to obstruction could elevate NGF levels independent of smooth muscle hypertrophy. This hypothesis was tested by examining neuronal size and NGF content in bladders of diuresed animals. Although voiding frequency in diuresed rats rose to the same extent as in urethral-ligated animals (Fig. 5), bladder weight slightly increased and neuronal areas remained $65 \%$ less than obstructed rats at $6 \mathrm{wk}$. NGF content of bladders from diuresed animals was only 2.5 times greater than controls compared to the 11 fold increase (per organ) seen with obstruction. Furthermore, the increase in voiding frequency in the autoimmune group was not associated with the same degree of neuronal enlargement as in obstructed animals (Fig. 3). These findings indicate that increased bladder activity is not the primary stimulus for neural plasticity and alterations in NGF. Rather, increased activity reflects other changes in the function of the bladder and its innervation that result from obstruction. None of the animals in this study were incontinent and the increased voiding frequency in diuresed animals was accounted for by increased water intake.

Denervation of smooth muscle or inflammation may alter NGF content (24). NGF content of smooth muscles in the iris and vas deferens varies with qualitative differences in neural input. NGF content in this situation represents the net balance

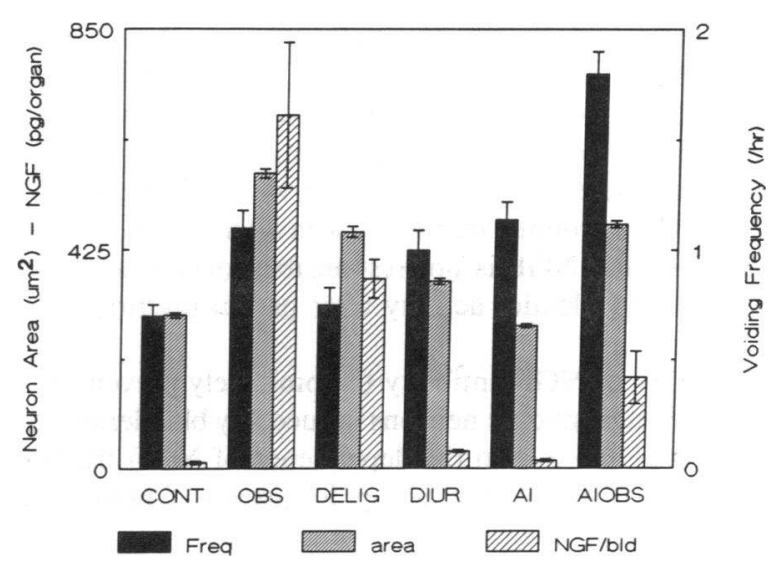

Figure 5. Nerve growth factor content of bladder after experimental manipulations. Urinary frequency (solid bars; $\mathbf{h}^{-1}$ ), bladder neuron size (grey bars; $\mu \mathrm{m}^{2}$ ) and NGF content (hatched bars; pg/organ) for eight control (CONT) animals ( 750 cells); six animals obstructed $(O B S)$ for $6 \mathrm{wk}$ ( 750 cells); six rats obstructed for $6 \mathrm{wk}$, then deligated (DELIG) for $6 \mathrm{wk}$ ( 630 cells); eight diuresed (DIUR) rats ( 710 cells), eight autoimmune animals (AI) (611 cells), and six rats made autoimmune then obstructed for 6 wk (AIOBS) ( 715 cells). NGF content and neuronal size increased in all obstructed groups $(P<0.001)$ compared to control. Autoimmunity alone caused a significant $(P$ $<0.05$ ) decrease in mean neuronal area compared with control, whereas mean area in AIOBS group was significantly $(P<0.001)$ lower than in OBS animals. 
between synthesis by target tissues and uptake by axons. Acute obstruction elicits a relative denervation of the bladder because ultrastructural studies show axonal degeneration with subsequent reinnervation of smooth muscle (20). Alternatively, inflammatory mediators including IL-1 can stimulate production of NGF. However, preliminary data in our laboratory show that surgical denervation of the bladder or intravesical instillation of chemical irritants do not raise NGF levels to the degree observed with obstruction (25). Taken together these studies suggest that increased NGF production is closely linked to the mechanisms underlying smooth muscle hypertrophy. This hypothesis is strengthened by the finding that cultured smooth muscle cells contain mRNA coding for NGF and synthesize NGF (26).

Questions remain as to the molecular basis for growth factor regulation. Recent circumstantial evidence has linked elevations in intracellular calcium and subsequent expression of protooncogenes with cardiac hypertrophy (27). Enhanced expression of protooncogenes such as c-fos, c-jun, and c-myc have also been associated with increases in NGF production by glia and fibroblasts (28). These secondary alterations in gene expression may contribute to an increased capacity of the cell for synthesis of contractile proteins and to the activation of genes responsible for the manufacture of a wide range of growth factors such as NGF.

We attempted to link changes in NGF with alterations in target organ function. However, the use of voiding frequency to assess bladder activity is complicated by the complex nature of factors governing micturition. The increased residual volume that occurs after obstruction can be associated with overflow incontinence, the presence of which would reduce the usefulness of measuring voiding frequency. However, none of the animals in this study were incontinent. Episodic voiding with periods of continence was observed. The AI rats voided more frequently than control rats, and AI did not prevent the rise in voiding frequency after obstruction. Endogenous NGF antibody could have increased urine output, enhanced smooth muscle activity or excitatory parasympathetic pathways regulating voiding, or reduced sympathetic inhibition of micturition pathways. These factors may explain why AI did not prevent the increase in voiding frequency seen after obstruction. It is also possible that NGF is not responsible for the development of increased bladder activity after partial urethral ligation.

The inability of NGF antibody to completely prevent hypertrophy of postganglionic neurons induced by bladder outlet obstruction could be due to the dependence of NGF-insensitive neurons on other trophic factors for growth. Cholinergic neurons in the pelvic ganglion provide excitatory input to the smooth muscle of the bladder (29). Therefore, trophic factors which influence the growth and survival of peripheral cholinergic cells such as ciliary neurotrophic factor (30), brain-derived neurotrophic factor (31), and basic fibroblast growth factor (32) may be responsible for the hypertrophy of NGF-insensitive neurons. It is also possible that the antibody identified by our dorsal root ganglion bioassay technique was ineffective or its access to bladder neurons limited in vivo. However, the reduction in mean size of MPG neurons in AI animals argues that the antibody was effective and its access sufficient. These data do not establish NGF as the sole mediator of the postobstructive neural plasticity. NGF may be operating alone or it may be permissive for secondary events. The ability of autoim- munity to NGF to prevent some of the neural growth does not distinquish between a primary and permissive role for the neurotrophin. However, this more complex relationship lacks precedent and the more parsimonious conclusion is a direct effect of NGF.

Another goal of this investigation was to evaluate the reversibility of neurotrophic and functional alterations elicited by obstruction. Deligation of obstructed animals was associated with a return of voiding frequency to control levels and a fall in NGF. However, NGF and neuronal areas remained above control values. Because bladder weights remained elevated it is possible that obstruction was still present. It is also possible that the changes induced by obstruction would have reversed completely if experiments were conducted beyond $6 \mathrm{wk}$, because it cannot be assumed that the rate of reversal is the same as the rate of onset. Another explanation is that neural changes which occur in response to $6 \mathrm{wk}$ of obstruction are not completely reversible. It is tempting to speculate that an incomplete reversal in neural plasticity may explain the continued symptoms and bladder dysfunction in patients after surgical relief of obstruction.

NGF and trophic factors may participate in other changes associated with bladder hypertrophy. In addition to regulating the growth and survival of postganglionic neurons in the peripheral nervous system, NGF also affects sensory neurons. Afferent neurons supplying the bladder demonstrate significant plasticity in response to obstruction (5-7). Similar to neurons in the pelvic ganglia, afferent neurons in the $L_{6}$ and $S_{1}$ DRG projecting to the rat bladder undergo hypertrophy. Furthermore, a $60 \%$ increase in the area of WGA-HRP labeled afferent projections to the intermediolateral cell column from obstructed bladders is observed (5-7). One mechanism for these morphologic changes may be an increased availability and retrograde transport of NGF from the hypertrophied bladder.

Neural regulation of micturition involves a complex series of events involving central and peripheral autonomic pathways (26). Electrophysiological analysis of normal rats and cats has shown that micturition is regulated by a supraspinal reflex relayed through the pons. A second reflex pathway, termed a spinal reflex, develops in spinal cord-transected animals with the resumption of micturition. It is of interest that obstructed rats consistently demonstrate electrophysiological evidence of a spinal reflex in addition to the supraspinal reflex (3). Thus, increased NGF in obstructed bladders may produce a functional plasticity through altered circuitry or patterns of connectivity within the sacral spinal cord. These changes in afferent pathways and a spinal reflex could in turn contribute increases in voiding frequency in our model or to the sensory symptoms and bladder hyperactivity observed in individuals with bladder outlet obstruction. A positive feedback system may exist whereby the central nervous system responds to bladder hypertrophy by reinforcing pathways responsible for voiding. It has been postulated that the functional obstruction that occurs after spinal cord transection produces bladder hypertrophy which thereby leads to the emergence of a spinal micturition reflex (7). This reflex is required for the return of voiding in paraplegics after an initial period of detrusor areflexia.

Our data linking bladder hypertrophy, neuronal enlargement and changes in organ function with NGF content offers an important new mechanism to explain trophic interactions between a target and its nerve supply in the adult. Although the partial urethral ligation model in the rat differs in some respects 
from pathology exhibited in patients with lower urinary tract obstruction, preliminary observations in humans raise the possibility that changes in NGF may participate in clinical disorders associated with organ enlargement or smooth muscle hypertrophy. If neurotrophic substances act as molecular signals that initiate the dynamic interactions between an organ and its innervation, the possibility exists that manipulation of growth factors or their receptors could provide a useful method of therapeutic intervention for certain disease processes.

In summary, the data presented suggest a novel mechanism making a contribution to obstruction-induced changes in efferent pathways to the bladder. An outlet obstruction causes bladder smooth muscle to respond to the increased functional demand with hypertrophy and/or hyperplasia. Concurrent with these muscle growth responses, neurotrophic factors, including NGF, are produced in greater amounts by the bladder muscle. Neurons innervating the bladder acquire the increased neurotrophins and themselves respond with growth. The neural growth is postulated to affect the reflex function, perhaps contributing to lowered reflex thresholds and altered visceromotor function.

\section{Acknowledgments}

Mouse NGF was a generous gift of Dr. E. M. Johnson, Washington University, St. Louis, MO. The authors would like to gratefully acknowledge the work of Bobra Slaughter in the preparation of this manuscript.

Work supported by National Institutes of Health grants $1 \mathrm{~K} 11$ DK1732-04 and R29NS 28566-01 and by grant-in-aid 901321 from the American Heart Association.

\section{References}

1. Holtgrewe, H. L., W. K. Mebust, J. B. Dowd, A. Cockett, P. C. Peters, and C. Proctor. 1989. Transurethral prostatectomy: practice aspects of the dominant operation in American urology. J. Urol. 141:248-253.

2. Anderssen, J. T. 1976. Detrusor hyperreflexia in benign infravesical obstruction. A cystometric study. J. Urol. 115:532-536.

3. Steers, W. D., and W. C. de Groat. 1988. Effect of bladder outlet obstruction on micturition reflex pathways in the rat. J. Urol. 140:864-871.

4. Steers, W. D., J. Ciambotti, S. Erdman, and W. C. de Groat. 1990. Morphological plasticity in efferent pathways to the urinary bladder of the rat following urethral obstruction. J. Neurosci. 10:1943-1951.

5. Steers, W. D. 1990. Neuroplasticity secondary to infravesical obstruction. Neurourol. Urodyn. 9:559-561. (Abstr.)

6. Steers, W. D., J. Ciambotti, B. Etzel, S. Erdman, and W. C. de Groat. 1988. Neural plasticity in afferent pathways to the urinary bladder following urethral obstruction in the rat. J. Comp. Neurol. In press.

7. de Groat, W. D., M. Kawatani, T. Hisamitsu, C.-L. Chenc, C.-P. Ma, K Thor, W. Steers, and J. R. Roppolo. 1990. Mechanisms underlying the recovery of urinary bladder function following spinal cord injury. J. Auton. Nerv. Syst. 30:571-578.

8. Purves, D., W. D. Snider, and J. T. Voyvodic. 1988. Trophic regulation of nerve cell morphology and innervation in the autonomic nervous system. Nature (Lond.). 336:123-128

9. Voyvodic, J. F. 1989. Peripheral target regulation of dendritic geometry in the rat superior cervical ganglion. J. Neurosci. 9:1997-2010.

10. Johnson, E. M., and I. A. Ferguson. 1989. Nerve growth factor. In Nerve Growth Factors. R. A. Rush, editor. John Wiley \& Sons, New York. 213-240.

11. Uvelius, B., and A. Mattiasson. 1984. Collagen content in the rat urinary bladder subjected to infravesical obstruction. J. Urol. 132:587-590.

12. Gorin, P. D., and E. M. Johnson. 1980. Effects of long-term nerve growth factor deprivation on the nervous system of the adult rat: an experimental autoimmune approach. Brain Res. 198:27-42.

13. Lowry, O. N., N. J. Rosebrough, A. L. Farr, and R. J. Randall. 1951. Protein measurement with the folin phenol reagent. J. Biol. Chem. 193:265-273.

14. Meghji, P., J. B. Tuttle, and R. Rubio. 1989. Adenosine formation and release by embryonic chick neurons and glia in cell culture. J. Neurochem. 53:1852-1860.

15. Westkamp, G., and U. Otten. 1987. An enzyme-linked immunoassay for nerve growth factor (NGF): a tool for studying regulatory mechanisms involved in NGF production in brain and in peripheral tissues. J. Neurochem. 48:17791786.

16. Hellweg, R., C. Hoch, and H. D. Hartung. 1989. An improved rapid and highly sensitive enzyme immunoassay for nerve growth factor. Tech. J. Cell. Mol. Biol. 1:43-48.

17. Earlam, R. J. 1971. Ganglion cell changes in experimental stenosis of the gut. Gut. 12:393-398.

18. Gabella, G. 1984. Size of neurons among glial cells in the intramural ganglia of the hypertrophic intestine of the guinea pig. J. Neurocytol. 13:73-84.

19. Elbadawi, A. S., S. Meyer, B. Malkowitz, A. Wein, R. Levin, and M. A. Atta. 1988. Effect of short-term partial bladder outlet obstruction on rabbit detrusor: an ultrastructural study. Neurourol. Urodyn. 8:89-116.

20. Creedon, D. J., W. D. Steers, and J. B. Tuttle. 1989. Potential role for NGF in neuronal plasticity after bladder obstruction. Soc. Neurosci. Abstr. 16:307. (Abstr.)

21. Steers, W. D., and J. B. Tuttle. 1990. Dissociated cell culture of adult rat pelvic ganglion. Soc. Neurosci. Abstr. 15:300. (Abstr.)

22. Keast, J. J., and W. C. de Groat. 1989. Immunocytochemical characterization of pelvic neurons which project to the bladder, colon or penis in rats: $J$. Comp. Neurol. 288:287-400.

23. Bjerre, B., and E. Rosengren. 1974. Effects of nerve growth factor and its antiserum on axonal regeneration of short adrenergic neurons in the male mouse. Cell Tissue Res. 150:9299-322.

24. Ebendal, T., L. Olson, and A. Seiger. 1983. The level of nerve growth factor as a function of innervation. Exp. Cell Res. 148:311-317.

25. Albo, M., L. Nataluk, W. Steers, and J. Tuttle. 1991. Morphological, functional and neurotrophic alterations in partially decentralized and denervated bladders. J. Urol. 145:308A. (Abstr.)

26. Creedon, D. J., W. D. Steers, and J. B. Tuttle. 1988. Cultured vascular smooth muscle cells provide NGF-dependent neuronal survival. Soc. Neurosci. Abstr. 14:680. (Abstr.)

27. Marban, E., and K. Yukihiro. 1990. Cell calcium, oncogenes, and hypertrophy. Hypertension (Dallas). 15:652-658.

28. Hengerer, B., D. Lindholm, R. Heumann, U. Ruther, E. F. Wagner, and H. Thoenen. 1990. Lesion-induced increase in nerve growth factor mRNA is mediated by c-fos. Proc. Natl. Acad. Sci. USA 87:3899-3903.

29. de Groat, W. D., and W. D. Steers. 1990. Autonomic regulation of the urinary bladder and sex organs. In Central Regulation of Autonomic Function. R. M. Spyer and A. Loewy, editors. Oxford University Press, Oxford. 310-333.

30. Tuttle, J. T., and G. Pilar. 1986. Target tissue influences on cholinergic development of parasympathetic neurons. Dynamics of cholinergic function. Adv. Behav. Biol. 30:879-890.

31. Barde, Y.-A., E. Edgar, and H. Thoenen. 1982. Purification of a new neurotrophic factor from mammalian brain. EMBO (Eur. Mol. Biol. Organ.) J. 1:533-549.

32. Vaca, K., S. S. Stewart, and S. H. Apel. 1989. Identification of basic fibroblast growth factor as a cholinergic growth factor from human muscle. $J$. Neurosci. Res. 23:55-63. 